\title{
OPEN Test-retest reliability of laser evoked pain perception and fMRI BOLD responses
}

\author{
Yanzhi $\mathrm{Bi}^{1,2}$, Xin $\mathrm{Hou}^{1,2}$, Jiahui Zhong ${ }^{3} \& \mathrm{Li} \mathrm{Hu}^{1,2 \bowtie}$
}

Pain perception is a subjective experience and highly variable across time. Brain responses evoked by nociceptive stimuli are highly associated with pain perception and also showed considerable variability. To date, the test-retest reliability of laser-evoked pain perception and its associated brain responses across sessions remain unclear. Here, an experiment with a within-subject repeatedmeasures design was performed in 22 healthy volunteers. Radiant-heat laser stimuli were delivered on subjects' left-hand dorsum in two sessions separated by 1-5 days. We observed that laser-evoked pain perception was significantly declined across sessions, coupled with decreased brain responses in the bilateral primary somatosensory cortex (S1), right primary motor cortex, supplementary motor area, and middle cingulate cortex. Intraclass correlation coefficients between the two sessions showed "fair" to "moderate" test-retest reliability for pain perception and brain responses. Additionally, we observed lower resting-state brain activity in the right $\mathrm{S} 1$ and lower resting-state functional connectivity between right $\mathrm{S} 1$ and dorsolateral prefrontal cortex in the second session than the first session. Altogether, being possibly influenced by changes of baseline mental state, laser-evoked pain perception and brain responses showed considerable across-session variability. This phenomenon should be considered when designing experiments for laboratory studies and evaluating pain abnormalities in clinical practice.

Blood oxygenation level-dependent functional magnetic resonance imaging (BOLD fMRI) can measure human brain activity objectively and enable a deep understanding of neural processing mechanisms. With this technique, the function of pain pathway structures within the central nervous system in states of acute or chronic pain ${ }^{1-6}$, during therapeutic and psychological pain intervention $s^{3,7-9}$ has been extensively investigated. Recently, accumulating studies suggested that fMRI could be used as a technique to elucidate objective biomarkers for the diagnosis of pain ${ }^{10,11}$ as the magnitude of neural responses and self-report pain ratings are highly correlated in many situations ${ }^{12}$.

However, pain perception is a very subjective experience, and self-report of pain perception reflects a complex mix of physiological and psychological processes, including nociception, emotion, decision making, selfawareness, social cognition, and communicative tendencies ${ }^{13}$. It is important to note that, even in the same condition, pain experience is not static over time within one individual, and pain perception can fluctuate from time to time ${ }^{14}$. Brief painful heat pulses, as generated by infrared laser stimulator, have been adopted as a selective way to activate nociceptive $\mathrm{A} \delta$ - and C-fiber afferents and have been widely used to assess the function of the nociceptive system in humans ${ }^{15,16}$. It is well known that radiant-heat laser stimuli could induce pure pain (without tactile sensation) and robust BOLD activation and deactivation in multiple brain structures, which are responsible for the processing of the sensory, cognitive, and affective components of pain ${ }^{17}$. However, few studies have examined whether the pain perception evoked by radiant-heat laser stimuli and the associated BOLD responses remain stable over time. In other words, the test-retest reliability of laser-evoked pain perception and BOLD responses remains unassessed.

To address this issue, an experiment with a within-subject repeated-measures design was performed in 22 healthy volunteers. Radiant-heat laser stimuli were delivered on the dorsum of subjects' left hand to evoke pain perception and BOLD responses in two different sessions, which were separated by $1-5$ days. With both behavioral and BOLD fMRI data (task fMRI and resting-state fMRI), we assessed (1) whether laser-evoked pain perception (i.e., pain threshold and pain ratings) and BOLD responses were significantly different between the

${ }^{1}$ CAS Key Laboratory of Mental Health, Institute of Psychology, Chinese Academy of Sciences, Beijing 100101, China. ${ }^{2}$ Department of Psychology, University of Chinese Academy of Sciences, Beijing 100101, China. ${ }^{3}$ Research Center of Brain and Cognitive Neuroscience, Liaoning Normal University, Dalian 116029, China. ${ }^{\varpi}$ email: huli@ psych.ac.cn 


\begin{tabular}{|l|c|l|l|l|l|}
\hline \multirow{2}{*}{ Variables } & \multicolumn{2}{l}{ T1 } & \multicolumn{2}{l|}{ T2 } & \multirow{2}{*}{$\boldsymbol{p}$ value } \\
\cline { 2 - 6 } & Mean & SD & Mean & SD & \\
\hline Age, in year & 26.73 & 3.37 & & & \\
\hline Education, in year & 17.41 & 2.04 & & & \\
\hline BDI & 2.73 & 4.1 & & & \\
\hline SAI & 32.86 & 8.64 & 32.64 & 10.43 & 0.82 \\
\hline TAI & 38.23 & 8.54 & & & \\
\hline Skin temperature, in ${ }^{\circ} \mathrm{C}$ & 31.63 & 0.97 & 31.46 & 0.92 & 0.334 \\
\hline Pain-related & & & & & \\
\hline PSQ & 4.42 & 1.30 & & & \\
\hline Pain threshold, in J & 2.68 & 0.33 & 2.84 & 0.43 & 0.04 \\
\hline Pain rating & 5.12 & 1.14 & 4.59 & 0.95 & 0.004 \\
\hline
\end{tabular}

Table 1. Demographics and behavioral results. BDI Beck Depression Inventory, SAI State Anxiety Inventory, TAI Trait Anxiety Inventory, PSQ pain sensitivity questionnaire, SD standard deviation.

two sessions; (2) the test-retest reliability of laser-evoked pain perception and BOLD responses in healthy subjects; (3) the possible influence of the changes of resting-state brain activity on the modulation of laser-evoked pain perception and BOLD responses.

\section{Results}

Behavioral results. Twenty-two healthy, right-handed, and pain-free male volunteers (mean age \pm standard deviation: $26.73 \pm 3.37$ years, mean education years \pm standard deviation: $17.41 \pm 2.04$ years) were recruited in this study. Each subject participated in two sessions (session 1: T1; session 2: T2) separated by 1-5 days (1.82 \pm 1.12 days). All subjects completed the two experiment sessions. As compared with T1 session, the pain threshold was significantly larger (T1: $2.68 \pm 0.33, \mathrm{~T} 2: 2.84 \pm 0.43, p=0.04$; Table 1), and pain ratings to laser stimuli were significantly lower (T1: $5.12 \pm 1.14$, T2: $4.59 \pm 0.95, p=0.004$; Table 1 ) in T2 session. The skin temperature (T1: $31.63 \pm 0.97, \mathrm{~T} 2: 31.46 \pm 0.92, p=0.334$; Table 1 ) and state anxiety (State Anxiety Inventory, SAI; T1: $32.86 \pm 8.64$, T2: $32.64 \pm 10.43, p=0.82$; Table 1 ) were not significantly different between the two sessions, which excluded the possible influences of skin temperature and state anxiety on the changes of pain perception between the two sessions.

Brain responses to nociceptive stimuli. For both sessions, nociceptive stimuli elicited significant activations in a wide array of subcortical and cortical brain regions, including the periaqueductal gray, thalamus, primary (S1) and secondary (S2) somatosensory cortices, insula, and the anterior cingulate cortex (ACC) (voxelwise $p<0.005$ and cluster-corrected at family wise error (FWE) of $p<0.05$; Fig. 1a, b). These brain activation patterns strongly resemble the findings previously described ${ }^{18-21}$. The paired-sample t-test revealed that the magnitudes of brain activations were significantly smaller in bilateral S1, primary motor cortex (M1), supplementary motor area (SMA), and middle cingulate cortex (MCC) in T2 session than in T1 session (voxel-wise $p<0.005$ and cluster-corrected at FWE of $p<0.05$; Fig. 1c). These results showed that the decrease in pain ratings was accompanied by a reduced BOLD response in brain regions responsible for nociceptive information processing.

Test-retest reliability. For pain threshold, the intraclass correlation coefficient (ICC) between the two sessions showed fair-reliability (ICC $=0.56$; Table 2 ), while the ICC for pain ratings to nociceptive laser stimuli demonstrated moderate-reliability ( ICC $=0.657$; Table 2). For both mean BOLD responses (the clusters showing significant differences between the two sessions) and mean region of interest (ROI) BOLD responses (ROIs in the "Pain Matrix"), ICCs also showed fair-to-moderate test-retest reliability (Table 2).

Spontaneous brain activity and resting-state functional connectivity. Decreased spontaneous brain activity (fractional amplitude of low-frequency fluctuations, fALFF) of the right $\mathrm{S} 1$ was observed in T2 session as compared with T1 session (paired-sample t-test, voxel $\mathrm{z}$ value $>2.3$, cluster significance: $p<0.05$, gaussian random field (GRF) corrected, two-tailed; Fig. 2a). When selecting the right S1 as the ROI for resting-state functional connectivity analysis, we observed that the right $\mathrm{S} 1$ exhibited weaker resting-state functional connectivity with the bilateral dorsolateral prefrontal cortex in T2 session as compared with T1 session (paired-sample t-test, voxel $\mathrm{z}$ value $>2.3$, cluster significance: $p<0.05$, GRF corrected, two-tailed; Fig. $2 b$ ).

\section{Discussion}

As compared with T1 session, declined laser-evoked pain perception coupled with decreased brain responses in bilateral S1, M1, SMA, and MCC were observed in T2 session that was performed 1-5 days later. Test-retest reliability analysis revealed that the ICCs of both pain perception and brain responses elicited by nociceptive laser stimuli were $0.434-0.751$, which represented fair-to-moderate reliability. When evaluating the possible effects of baseline spontaneous brain activity on the changes of laser-evoked pain perception and brain responses, we found decreased spontaneous brain activity in the right $S 1$ and decreased resting-state functional connectivity between the right S1 and bilateral dorsolateral prefrontal cortex in T2 session as compared with T1 session. 


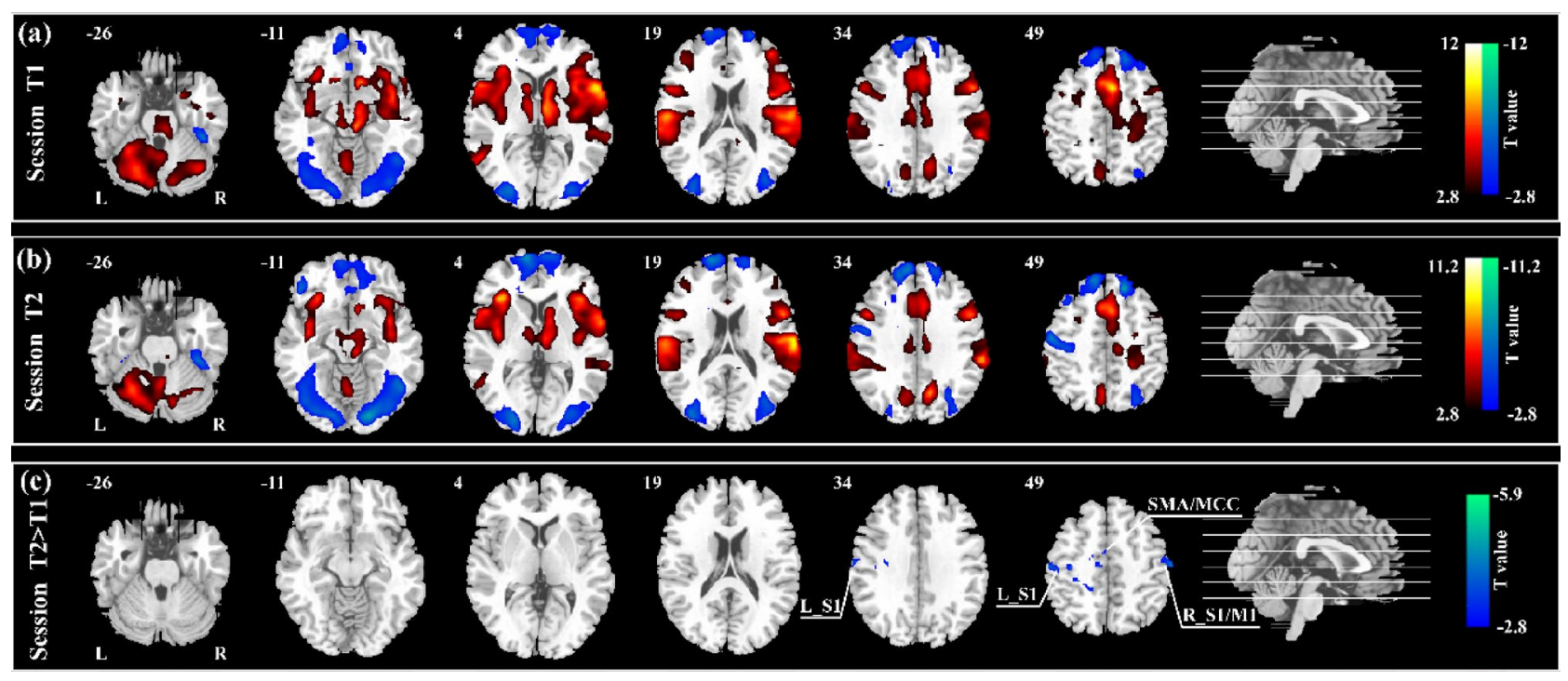

Figure 1. Brain responses to nociceptive laser stimuli. For both $\mathrm{T} 1$ session (a) and $\mathrm{T} 2$ session (b), nociceptive stimuli elicited significant brain responses in the periaqueductal gray, thalamus, primary somatosensory cortex (S1), secondary somatosensory cortex, insula, and anterior cingulate cortex. (c) Laser-evoked brain responses were significantly smaller in bilateral S1, right primary motor cortex (M1), supplementary motor area (SMA), and middle cingulate cortex (MCC) during T2 session than T1 session. $L$ left hemisphere; $R$ right hemisphere. The resulting t-statistic maps were thresholded at $p<0.005$, cluster-corrected at family wise error of $p<0.05$. The images were created by using the MRIcron software (v1.0.20190902, https://www.nitrc.org/projects/mricron).

\begin{tabular}{|c|c|c|c|c|c|}
\hline & ICC & F value & $p$ value & CI lower & CI upper \\
\hline \multicolumn{6}{|l|}{ Pain perception } \\
\hline Pain threshold & 0.56 & 3.986 & 0.001 & 0.198 & 0.789 \\
\hline Pain rating & 0.657 & 6.445 & $<0.001$ & 0.233 & 0.854 \\
\hline \multicolumn{6}{|l|}{ Mean BOLD responses } \\
\hline Left S1 & .698 & 9.922 & $<0.001$ & .091 & .893 \\
\hline Right S1/M1 & .564 & 5.606 & $<0.001$ & .030 & .820 \\
\hline SMA/MCC & .685 & 8.901 & $<0.001$ & .113 & .884 \\
\hline \multicolumn{6}{|c|}{ Mean ROI BOLD responses } \\
\hline Left anterior insula & .549 & 3.395 & .004 & .177 & .784 \\
\hline Right anterior insula & .448 & 2.552 & .019 & .030 & .729 \\
\hline Left posterior insula & .574 & 3.636 & .002 & .211 & .798 \\
\hline Right posterior insula & .487 & 2.900 & .009 & .099 & .748 \\
\hline Left ACC & .696 & 5.621 & .000 & .404 & .860 \\
\hline Right ACC & .682 & 5.427 & .000 & .384 & .853 \\
\hline Left thalamus & .434 & 2.510 & .020 & .026 & .718 \\
\hline Right thalamus & .454 & 2.683 & .014 & .060 & .728 \\
\hline Left S1 & .661 & 6.689 & .000 & .224 & .858 \\
\hline Right S1 & .660 & 6.488 & .000 & .240 & .856 \\
\hline Left S2 & .684 & 5.529 & .000 & .387 & .854 \\
\hline Right S2 & .751 & 7.329 & .000 & .495 & .888 \\
\hline
\end{tabular}

Table 2. Intraclass correlation coefficients of pain perception and laser-evoked brain responses between the two sessions. The intraclass correlation coefficients (ICCs) were calculated using the single-rating, absoluteagreement, 2-way mixed model. 95\% confidence interval (CI) is the interval between CI lower and CI upper. ROI, region of interest; S1, primary somatosensory cortex; S2, secondary somatosensory cortex; M1, primary motor cortex; SMA, supplementary motor area; MCC, middle cingulate cortex; ACC, anterior cingulate cortex. Bonferroni correction, $p<0.025$ for pain perception analyses and $p<0.05 / 15$ for brain response analyses. 
(a)

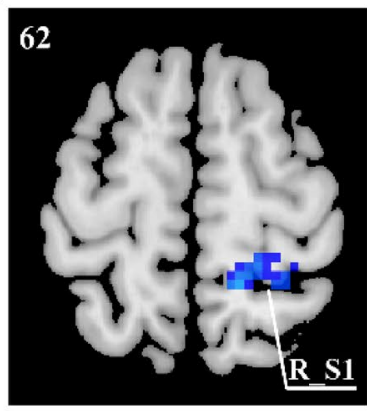

fALFF difference

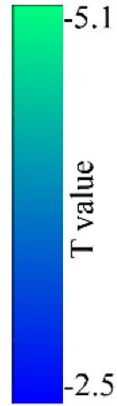

$-2.5$
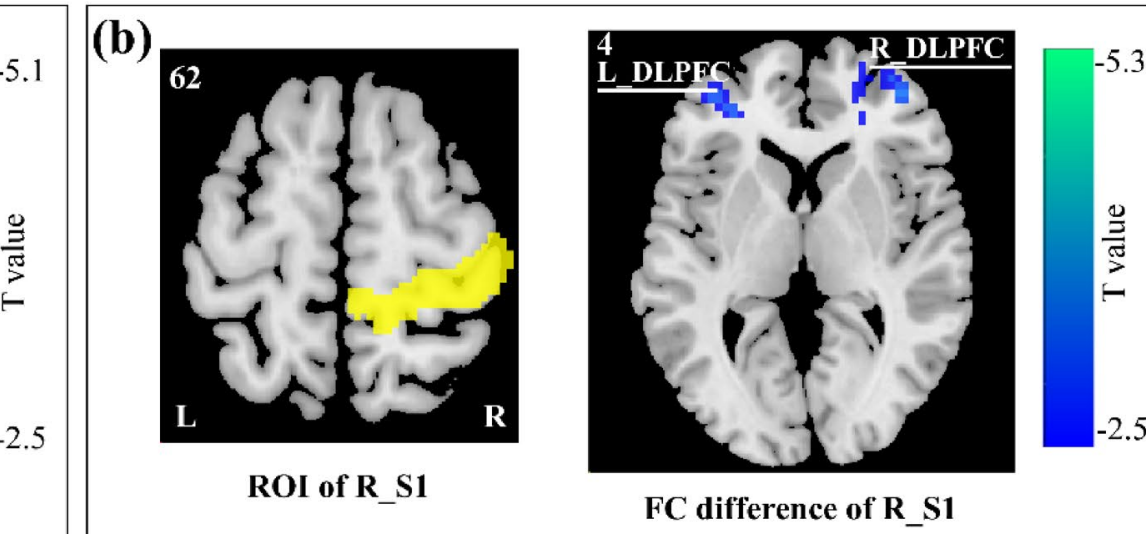

FC difference of $R \_S 1$

Figure 2. Differences in spontaneous brain activity and resting-state functional connectivity between the two sessions. (a) Decreased spontaneous brain activity (fALFF) of the right S1 was observed during T2 session as compared with $\mathrm{T} 1$ session. (b) When the right $\mathrm{S} 1$ was selected as the ROI, the right $\mathrm{S} 1$ showed decreased resting-state functional connectivity with the bilateral dorsolateral prefrontal cortex (DLPFC) during T2 session as compared with T1 session. $L$ left hemisphere, $R$ right hemisphere. The resulting t-statistic maps were corrected for multiple comparisons using the gaussian random field (GRF) theory with a voxel $\mathrm{z}$ value $>2.3$ and cluster $p$ value $<0.05$ (two-tailed correction). The images were created by using the Data Processing \& Analysis for (Resting-State) Brain Imaging (DPABI) software (DPABI_V5.0_201001, http://rfmri.org/dpabi) ${ }^{59}$.

Altogether, being possibly influenced by the modulation of baseline mental state, laser-evoked pain perception and brain responses showed considerable variability between different experiment sessions. This phenomenon should be considered when designing experiments for laboratory studies and evaluating pain abnormalities in clinical practice.

Recent studies showed that mildly noxious stimuli resulted in lower repeatability for experimental pain perception across different sessions than highly painful stimuli ${ }^{14,22}$ and that ratings of pain perception trended downward across sessions ${ }^{14,22}$. These observations were consistent with our findings that laser-evoked pain perception declined significantly from $\mathrm{T} 1$ session to T2 session when laser stimuli with a fixed stimulus intensity of $3.5 \mathrm{~J}$ elicited a mild-moderate painful sensation (Table 1). One possible explanation of this observation is the difficulty of the rating task for innocuous or mildly noxious stimuli as compared with highly painful stimuli ${ }^{14}$. In addition, the test-retest reliability of pain perception was fair-to-moderate (i.e., ICC $=0.56-0.657$, Table 2). By definition, ICC is the ratio of the between-subject variance to the total variance across repeated measures ${ }^{23}$. Any factors that decrease the between-subject variability or increase the within-subject variability will decrease test-retest reliability. In the present study, the declined pain perception from T1 session to T2 session would contribute to the increase of the within-subject variability, which ultimately resulted in decreased reliability of laser-evoked pain perception.

It should be noted that the results at the behavioral level were fully supported by the findings of laser-evoked brain responses: BOLD responses elicited by nociceptive laser stimuli were significantly decreased in bilateral S1, M1, SMA, and MCC across the two sessions (Fig. 1c). Due to the significant decrease of laser-evoked brain responses, the test-retest reliability was not very high, in the range from 0.434 to 0.751 , i.e., "fair" to "moderate" reliability. Since the magnitude of neural responses sampled using fMRI technique is capable of encoding subjective pain ratings in most practical situations ${ }^{12,24,25}$, our finding observed at the neural level suggested the reliability of the results obtained at the behavioral level.

It is well known that pain experience is highly variable and could be influenced by factors at different dimensions (e.g., physical, physiological, psychological, social, and cultural factors), which support a multidimensional framework for the modulation of the pain experience ${ }^{26}$. For instance, being stimulated by the same physical stimuli, pain perception would be different when the psychological state (e.g., anxiety level, the fearful level to the stimuli, and the attention level) of the subjects was changed ${ }^{3,9,27-29}$. In the present study, the psychological state of subjects could be different between the two sessions, as the subjects became more familiar with the experimental environment and the nociceptive stimuli in T2 session than in T1 session. It is be possible that with the adaptation to the experiment, the subjects are less anxious and less fearful to nociceptive laser stimuli, as such the attentional level to nociceptive stimuli and the stimulated site might be decreased in T2 session as compared with $\mathrm{T} 1$ session.

In line with the decreased brain responses in the $\mathrm{S} 1$, baseline spontaneous brain activity in the right $\mathrm{S} 1$ was also decreased from T1 session to T2 session (Fig. 2a). It is well recognized that the S1 is highly associated with the sensory-discriminative aspects of pain $^{30}$, including the localization and discrimination of stimulus intensity, and its activation is easily modulated by several psychological factors (e.g., anticipation and attention) ${ }^{31-33}$. Mennes and colleagues demonstrated that a region's task-evoked BOLD responses could be predicted by that region's spontaneous brain activity ${ }^{34}$. For this reason, the changes in baseline spontaneous brain activity in the S1 could influence the laser-evoked brain responses in this region, which may lead to the decrease in sensorydiscriminative processing of pain and the decrease in pain perception from T1 session to T2 session. On the other hand, S1 activation is highly modulated by cognitive factors that alter pain perception, including attention and 
previous experience ${ }^{31}$. With the adaption to the experimental environment and nociceptive stimuli, the focus of attention on nociceptive stimuli and the stimulated site was likely reduced in T2 session as compared with T1 session, which would contribute to the decrease of S1 activation and the decline of the self-report of pain perception.

Furthermore, resting-state functional connectivity between right S1 and bilateral dorsolateral prefrontal cortex was lower in T2 session as compared with T1 session (Fig. 2b). Previous studies demonstrated that the dorsolateral prefrontal cortex is generally related to the cognitive and attentional processing of painful $\operatorname{stimuli}^{35,36}$. Thus, the decreased functional connectivity between right $\mathrm{S} 1$ and the dorsolateral prefrontal cortex is likely related to the changed attentional state to nociceptive stimuli from T1 session to T2 session. Altogether, the decreased baseline functional connectivity might influence both laser-evoked pain perception and brain responses by allocating fewer attention resources to nociceptive stimuli.

Pain perception is a multidimensional experience that encompasses sensory-discriminative, affective-motivational, and cognitive-evaluative components ${ }^{37}$. As a consequence, pain perception and the associated brain responses are considerably variable and have fair-to-moderate reliability across experimental sessions. The variability between different experiment sessions of laser-evoked pain perception and brain responses could be related to the changes of baseline mental state that could be easily influenced by several physiological factors (e.g., anxiety level, the fearful level to the stimuli, and the attention level). Hence, assessing the possible effects of psychological state on pain perception and brain responses and monitoring the baseline brain activity are highly needed when designing experiments for laboratory studies and evaluating pain abnormalities in clinical practice. For instance, the across-session variability should be taken into consideration when (1) examining the test-retest reliability of pain perception from multiple experimental sessions; (2) assessing the habituation effect of pain using repeated painful stimulation over several days; and (3) evaluating the long-term analgesic effect of treatment strategies in clinical practice or basic research. Besides, evidence showed that structural MRI features (e.g., gray matter intensity/volume, cortical thickness, subcortical volume) are highly correlated with pain perception in both healthy subjects $s^{38,39}$ and chronic pain patients ${ }^{40-42}$. Therefore, investigating the relationship between structural MRI features and the test-retest reliability of pain perception or brain responses in future work would provide new insights into the neural mechanism of test-retest reliability of pain.

\section{Methods}

The experiment was approved by the ethical standards of the Institutional Review Board of the Institute of Psychology, Chinese Academy of Sciences. All procedures were performed in accordance with the Declaration of Helsinki. Written informed consent was obtained from each subject prior to data collection. Subjects were screened for DSM-IV axis I and II disorders using the Structured Clinical Interview for DSM-IV, and were excluded if they had any contraindications (e.g., brain lesions, non-removable metallic implants, claustrophobia, etc.) for MRI scanning, history of peripheral and neurological disorders, chronic pain disorders, or if they consumed medications and reported a history of alcoholism or drug abuse.

Pain sensitivity measurement. For each subject, pain sensitivity was assessed using the Pain Sensitivity Questionnaire $^{43}$, and pain threshold was evaluated using laser stimuli. Radiant-heat laser stimuli were generated by an infrared neodymium yttrium aluminum perovskite (Nd:YAP) laser with a wavelength of $1.34 \mu \mathrm{m}$ and a pulse duration of $4 \mathrm{~ms}$ (Electronical Engineering, Italy) ${ }^{44}$. At this wavelength and pulse duration, laser stimuli activate directly nociceptive terminals in the most superficial skin layers in a synchronized fashion ${ }^{45}$. A He-Ne laser pointed to the area to be stimulated. The laser pulse was transmitted via an optic fiber and focused by lenses to a spot with a diameter of $\sim 7 \mathrm{~mm}\left(\sim 38 \mathrm{~mm}^{2}\right)$. Laser pulses were delivered to a squared area $\left(4 \times 4 \mathrm{~cm}^{2}\right)$ on the dorsum of subjects' left hand (non-dominant hand). To prevent fatigue or sensitization of the nociceptors, the laser beam target was manually shifted by at least $1 \mathrm{~cm}$ in a random direction after each stimulus ${ }^{46-48}$. The method of limits was adopted for each subject to determine the pain threshold in each session: starting from $1.5 \mathrm{~J}$, the laser energy was increased in steps of $0.25 \mathrm{~J}$ until a rating of 4 out of 10 (i.e., the energy at which the subjects start to feel pain) was obtained on a numerical rating scale (NRS) ranging from 0 (no pain) to 10 (the worst pain imaginable $)^{44}$. Prior to the pain threshold measurement, the skin temperature of the left hand was recorded for each subject to monitor the possible effect of temperature on pain perception.

Experiment design. An experiment with a within-subject repeated-measures design was performed (Fig. 3a). Each subject participated in two sessions (session 1: T1; session 2: T2) separated by 1-5 days (1.82 \pm 1.12 days). In both T1 and T2 sessions, resting-state and task fMRI data were collected for each subject. To monitor subjects' mood state, depression was evaluated by Beck-Depression Inventory (BDI) ${ }^{49}$ in T1 session, and state and trait anxiety was evaluated by State-Trait Anxiety Inventory (STAI) ${ }^{50}$ in both sessions.

During the task fMRI scan, 30 laser pulses at fixed stimulus energy of $3.5 \mathrm{~J}$ were delivered to the dorsum of the left hand to elicit a painful pinprick sensation for each subject (Fig. 3b). Inter-stimulus interval varied randomly between 26 and $28 \mathrm{~s}$ with a rectangular distribution. Each trial started with a 4 -s white cross centered on the screen and followed by the laser stimulus (duration $=4 \mathrm{~ms}$ ). A visual cue presented $16 \mathrm{~s}$ after the laser stimulus prompted the subjects to rate the intensity of pain perception elicited by the laser stimulus within $5 \mathrm{~s}$ on the same NRS. The following trial started in $1-3 \mathrm{~s}$, and the duration of the whole task fMRI scan was $\sim 14$ min.

MRI data acquisition. The MRI data were collected using a 3.0-Telsa Magnetic Resonance Imaging (MRI) system with a standard birdcage head coil (Discovery MR 750; General Electric Healthcare; Milwaukee; Wisc.) at the MRI Research Center, Institute of Psychology, Chinese Academy of Sciences. T1-weighted structural image was acquired using the gradient echo (3D SPGR) sequence ( $1 \mathrm{~mm}^{3}$ isotropic voxel, field of view $\left.(\mathrm{FOV})=256 \times 256 \mathrm{~mm}^{2}\right)$. An echo-planar-imaging sequence (repetition time $=2000 \mathrm{~ms}$; echo time $=30 \mathrm{~ms}$; flip 

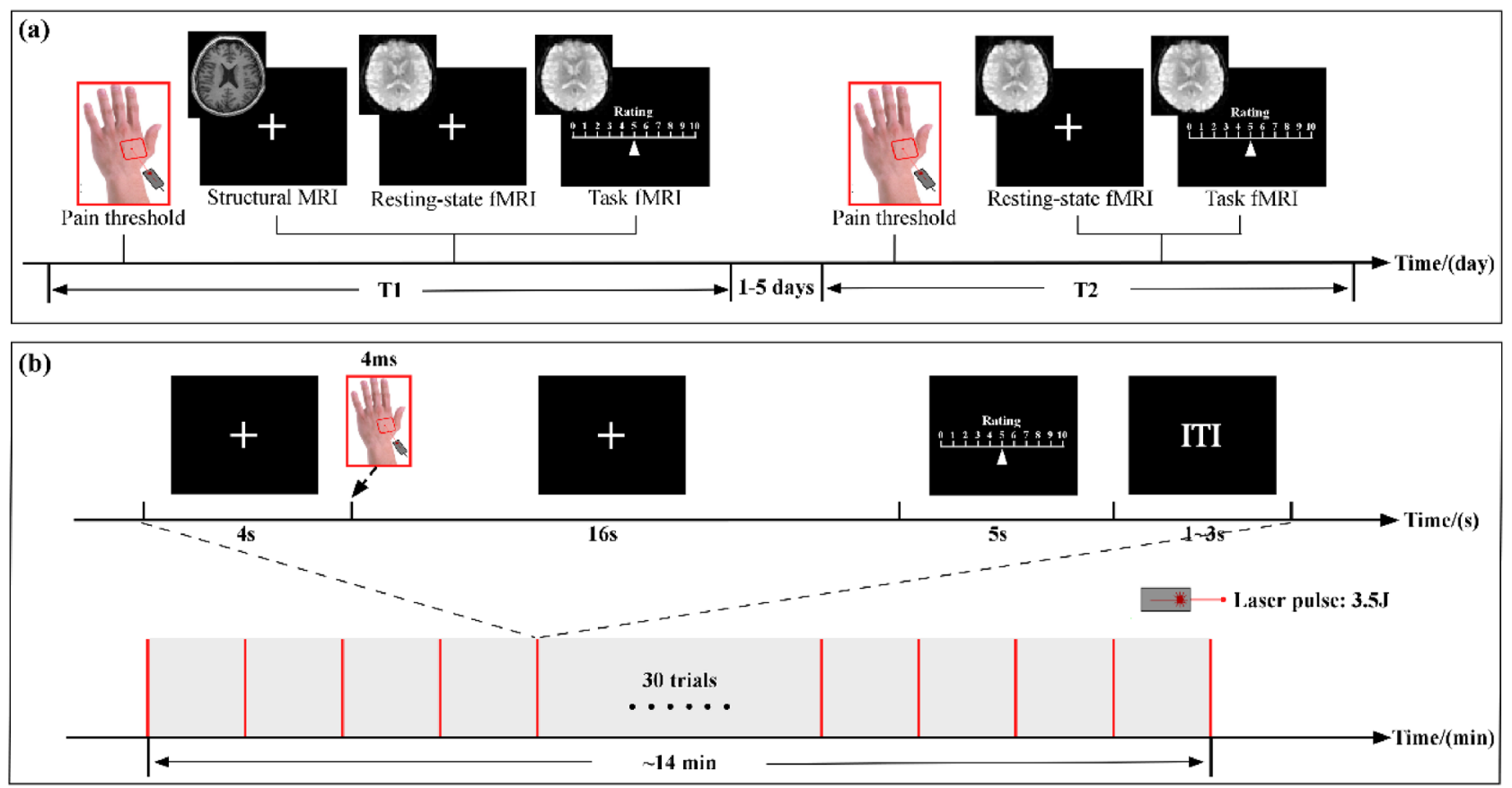

Figure 3. Experiment design and nociceptive stimulation. (a) Experimental design. (b) Task fMRI scan contained a single block of 30 trials with transient nociceptive stimuli. Each trial started with a 4-s fixation of the white cross-centered on the screen and followed by the delivery of a nociceptive stimulus. A visual cue presented $16 \mathrm{~s}$ after the nociceptive stimulus prompted the subjects to rate the perceived intensity within $5 \mathrm{~s}$ on the $0-10$ NRS. The inter-trial interval varied randomly between 1 and $3 \mathrm{~s}$. NRS, numerical rating scale. The MRI images were created by using the MRIcron software (v1.0.20190902, https://www.nitrc.org/projects/mricron).

angle $=90^{\circ} ; \mathrm{FOV}=64 \times 64 \mathrm{~mm}^{2} ;$ data matrix $=64 \times 64$; in-plane resolution $=3 \times 3 \mathrm{~mm}^{2} ;$ slice thickness $=3.5 \mathrm{~mm}$; slice spacing $=0.5 \mathrm{~mm}$ ) was used to acquire the resting-state functional data ( 300 volumes for resting-state fMRI session), and then the task fMRI data (409 volumes for task fMRI session). During the resting-state fMRI data acquisition, the screen presented a black fixation ' + ' in the center of the gray background. The patients were instructed to remain still with their eyes open, not to fall asleep, and not to think about anything in particular.

Task fMRI data processing. Task fMRI data were analyzed using the 12th edition of Statistical Parametric Mapping (SPM12, Welcome Department of Cognitive Neurology, London, UK, http://www.fil.ion.ucl.ac.uk/ $\mathrm{spm})$. The preprocessing analysis included the following procedures ${ }^{3}$ : discarding the first three volumes, slice timing correction, realignment and normalization to the EPI template with the resampling voxel size of $3 \times 3 \times 3$ $\mathrm{mm}^{3}$, spatial smoothing using a Gaussian kernel of $5 \mathrm{~mm}$ full width at half maximum (FWHM), and temporal filtering using a high pass filter and cut-off at $128 \mathrm{~s}$.

Statistical analysis of individual preprocessed imaging data was performed with first-level fixed-effects analyses using a general linear model. The regressor (nociceptive stimuli) was convolved with the canonical hemodynamic response function plus its temporal derivatives with realignment parameters (three translations and three rotations) included as regressors of no interest. For each subject, the contrast image of nociceptive stimuli was extracted to examine laser evoked BOLD responses within each group using a one-sample t-test. The resulted t-map of each session (T1 or T2) was thresholded at voxel-wise $p<0.005$ and cluster-corrected at FWE of $p<0.05$ at the whole-brain level. The differences in brain responses to nociceptive stimuli between sessions were assessed using a paired-sample t-test (voxel-wise $p<0.005$ and cluster-corrected at FWE of $p<0.05$ ).

Test-retest reliability. Test-retest reliability is the extent to which a dependent variable is consistent and free from error across sessions ${ }^{51}$. The ICC is a widely-used measure of test-retest reliability. The ICCs and their 95\% confidence intervals were calculated for pain threshold, pain rating, and laser-evoked BOLD responses between sessions 1 and 2 to evaluate their test-retest reliability using SPSS for Windows (Statistical Package for Social Sciences, Release 18.0, SPSS Inc., Chicago, IL, USA). In detail, ICCs of the absolute agreement were calculated using a two-way mixed model that provided a measure of consistency through a ratio of between-subject variance to the total variance ${ }^{52}$. For laser-evoked BOLD responses, mean BOLD data (defined in terms of parameter estimates) of clusters showing significant differences between the two sessions were extracted to calculate the ICCs. Besides, to assess the stability of brain activations in all regions of the "Pain Matrix"18-21, a series of ROIs, including the S1 and S2, thalamus, the insula, and the ACC, were selected. The ROIs of S1, S2, thalamus, and ACC were defined from the Harvard Oxford cortical and subcortical structural atlas ${ }^{53,54}$, which are population-based probability atlas in MNI 152 standard space. Four ROIs of the left and right anterior and posterior insula were defined as in our previous study ${ }^{55,56}$ using the analytic scripts downloaded via http://fcon_1000. 
projects.nitrc.org ${ }^{57}$. For each ROI, the mean BOLD response across all voxels within the ROI, defined in terms of parameter estimates, was extracted from each subject and each session. The degree of reliability of each value was then classified based on the conservative criteria described by Shrout ${ }^{58}$ : virtually no reliability $(0.00-0.10)$, slight-reliability $(0.11-0.40)$, fair-reliability $(0.41-0.60)$, moderate-reliability $(0.61-0.80)$, and substantial reliability (0.81-1.00). Bonferroni procedures was performed to correct for multiple comparisons $(p<0.025$ for pain perception analysis and $p<0.05 / 15$ for brain response analysis).

Resting-state fMRI data processing. Resting-state fMRI data were analyzed using Data Processing \& Analysis for (Resting-State) Brain Imaging (DPABI) software (DPABI_V5.0_201001, http://rfmri.org/dpabi) ${ }^{59}$. The first ten volumes were discarded for signal equilibrium and to allow the participants' adaptation to the scanning noise. Images were slice timing corrected and realigned. Structural images were segment into grey matter, white matter, and cerebrospinal fluid. White matter signal, cerebrospinal fluid signal, and head motion parameters were regressed as nuisance regressors from the corrected functional images. The processed functional data were then normalized to EPI template (resampling voxel size $=3 \times 3 \times 3 \mathrm{~mm}^{3}$ ), spatially smoothed using a Gaussian kernel of $5 \mathrm{~mm}$ FWHM, and detrended to remove the drifts and trends in the fMRI data.

Being defined as the ratio of the power spectrum of low-frequency fluctuations $(0.01-0.1 \mathrm{~Hz})$ to that of the entire frequency range $(0-0.25 \mathrm{~Hz})$, the fALFF were used to detect the intensity of regional spontaneous brain activity with high sensitivity and specificity ${ }^{60-63}$. To assess the spontaneous brain activity differences between the two sessions, fALFF was extracted and compared using a paired-sample t-test. Statistical maps were thresholded using the GRF theory correction procedure ${ }^{64,65}$, as implemented in DPABI ${ }^{59}$. The significance level was set at $p<0.05$ corrected for multiple comparisons (paired-sample t-test, voxel $\mathrm{z}$ value $>2.3$, cluster significance: $p<0.05$, GRF corrected, two-tailed).

Brain regions showed significant differences of fALFF between the two sessions were selected as ROIs for the functional connectivity analysis of resting-state fMRI data. For each ROI, the time series of spontaneous brain activity, which were averaged across all voxels within the ROI, were extracted in each subject, and individuallevel correlation maps of all voxels that were positively or negatively correlated with the averaged ROI time series were produced ${ }^{55,56}$. The resulting correlation maps were converted to Z-value maps using Fisher's r-to-Z transformation. A paired-sample t-test was performed to investigate the differences of resting-state functional connectivity for each ROI between the two sessions. Statistical maps were thresholded using the GRF theory correction procedure ${ }^{59,64,65}$, in which the significance level was set at $p<0.05$ corrected for multiple comparisons (voxel $\mathrm{z}$ value $>2.3$, cluster significance: $p<0.05$, GRF corrected, two-tailed).

Received: 1 October 2020; Accepted: 2 December 2020

Published online: 14 January 2021

\section{References}

1. Li, H. et al. Deficits in ascending and descending pain modulation pathways in patients with postherpetic neuralgia. NeuroImage 221, 117186. https://doi.org/10.1016/j.neuroimage.2020.117186 (2020).

2. Zhou, L. et al. A modality-specific dysfunction of pain processing in schizophrenia. Hum. Brain Mapp. 41, 1738-1753. https://doi. org/10.1002/hbm.24906 (2020).

3. Tu, Y., Bi, Y., Zhang, L., Wei, H. \& Hu, L. Mesocorticolimbic pathways encode cue-based expectancy effects on pain. J. Neurosci. 40, 382-394. https://doi.org/10.1523/jneurosci.1082-19.2019 (2019).

4. Hu, L. et al. The primary somatosensory cortex and the insula contribute differently to the processing of transient and sustained nociceptive and non-nociceptive somatosensory inputs. Hum. Brain Mapp. 36, 4346-4360. https://doi.org/10.1002/hbm.22922 (2015).

5. von Deneen, K. M., Zhao, L. \& Liu, J. Individual differences of maladaptive brain changes in migraine and their relationship with differential effectiveness of treatments. Brain Sci. Adv. 5, 239-255. https://doi.org/10.26599/BSA.2019.9050021 (2019).

6. Su, Q., Song, Y., Zhao, R. \& Liang, M. A review on the ongoing quest for a pain signature in the human brain. Brain Sci. Adv. 5, 274-287. https://doi.org/10.26599/BSA.2019.9050024 (2019).

7. Cao, J. et al. Analgesic effects evoked by real and imagined acupuncture: A neuroimaging study. Cereb. Cortex 29, 3220-3231. https ://doi.org/10.1093/cercor/bhy190 (2019).

8. Tu, Y. et al. Multivariate resting-state functional connectivity predicts responses to real and sham acupuncture treatment in chronic low back pain. NeuroImage Clin. 23, 101885. https://doi.org/10.1016/j.nicl.2019.101885 (2019).

9. Zhang, L., Lu, X., Bi, Y. \& Hu, L. Pavlov's pain: The effect of classical conditioning on pain perception and its clinical implications. Curr. Pain Headache Rep. 23, 19. https://doi.org/10.1007/s11916-019-0766-0 (2019).

10. Tu, Y. et al. An fMRI-based neural marker for migraine without aura. Neurology 94, e741-e751. https://doi.org/10.1212/WNL.00000 $00000008962(2020)$.

11. Brodersen, K. H. et al. Decoding the perception of pain from fMRI using multivariate pattern analysis. Neuroimage 63, 1162-1170. https://doi.org/10.1016/j.neuroimage.2012.08.035 (2012).

12. Robinson, M. E., Staud, R. \& Price, D. D. Pain measurement and brain activity: Will neuroimages replace pain ratings? J. Pain 14, 323-327. https://doi.org/10.1016/j.jpain.2012.05.007 (2013).

13. Woo, C.-W. \& Wager, T. D. What reliability can and cannot tell us about pain report and pain neuroimaging. Pain 157, 511-513. https://doi.org/10.1097/j.pain.0000000000000546 (2016).

14. Quiton, R. L. \& Greenspan, J. D. Across-and within-session variability of ratings of painful contact heat stimuli. Pain ${ }^{\star} 137,245-256$. https://doi.org/10.1016/j.pain.2007.08.034 (2008).

15. Bromm, B. \& Treede, R. Nerve fibre discharges, cerebral potentials and sensations induced by $\mathrm{CO}_{2}$ laser stimulation. Hum. Neurobiol. 3, 33 (1984).

16. Plaghki, L. \& Mouraux, A. How do we selectively activate skin nociceptors with a high power infrared laser? Physiology and biophysics of laser stimulation. Neurophysiol. Clin. 33, 269-277. https://doi.org/10.1016/j.neucli.2003.10.003 (2003).

17. Peyron, R., Laurent, B. \& Garcia-Larrea, L. Functional imaging of brain responses to pain. A review and meta-analysis. Clin. Neurophysiol. 30, 263-288. https://doi.org/10.1016/s0987-7053(00)00227-6 (2000).

18. Iannetti, G. D. \& Mouraux, A. From the neuromatrix to the pain matrix (and back). Exp. Brain Res. 205, 1-12. https://doi. org/10.1007/s00221-010-2340-1 (2010). 
19. Legrain, V., Iannetti, G. D., Plaghki, L. \& Mouraux, A. The pain matrix reloaded: A salience detection system for the body. Prog. Neurobiol. 93, 111-124. https://doi.org/10.1016/j.pneurobio.2010.10.005 (2011).

20. Hu, L. \& Iannetti, G. D. Painful issues in pain prediction. Trends Neurosci. 39, 212-220. https://doi.org/10.1016/j.tins.2016.01.004 (2016).

21. Mouraux, A., Diukova, A., Lee, M. C., Wise, R. G. \& Iannetti, G. D. A multisensory investigation of the functional significance of the "pain matrix". Neuroimage 54, 2237-2249. https://doi.org/10.1016/j.neuroimage.2010.09.084 (2011).

22. Hayashi, K. et al. Regional differences of repeatability on visual analogue scale with experimental mechanical pain stimuli. Neurosci. Lett. 585, 67-71. https://doi.org/10.1016/j.neulet.2014.11.032 (2015).

23. Koo, T. K. \& Li, M. Y. A guideline of selecting and reporting intraclass correlation coefficients for reliability research. J. Chiropract. Med. 15, 155-163. https://doi.org/10.1016/j.jcm.2016.02.012 (2016).

24. Büchel, C. et al. Dissociable neural responses related to pain intensity, stimulus intensity, and stimulus awareness within the anterior cingulate cortex: A parametric single-trial laser functional magnetic resonance imaging study. J. Neurosci. 22, 970-976. https:// doi.org/10.1523/JNEUROSCI.22-03-00970.2002 (2002).

25. Coghill, R. C., McHaffie, J. G. \& Yen, Y.-F. Neural correlates of interindividual differences in the subjective experience of pain. Proc. Natl. Acad. Sci. 100, 8538-8542. https://doi.org/10.1073/pnas.1430684100 (2003).

26. Ahles, T. A., Blanchard, E. B. \& Ruckdeschel, J. C. The multidimensional nature of cancer-related pain. Pain 17, 277-288. https:// doi.org/10.1016/0304-3959(83)90100-8 (1983).

27. Zhou, L. et al. The influence of expectancy level and personal characteristics on placebo effects: Psychological underpinnings. Front. Psychiatry 10, 20. https://doi.org/10.3389/fpsyt.2019.00020 (2019).

28. Wei, H. et al. The influence of expectation on nondeceptive placebo and nocebo effects. Pain Res. Manag. https://doi. org/10.1155/2018/8459429 (2018).

29. Zhang, H., Zhou, L., Wei, H., Lu, X. \& Hu, L. The sustained influence of prior experience induced by social observation on placebo and nocebo responses. J. Pain Res. 10, 2769. https://doi.org/10.2147/JPR.S147970 (2017).

30. Hofbauer, R. K., Rainville, P., Duncan, G. H. \& Bushnell, M. C. Cortical representation of the sensory dimension of pain. J. Neurophysiol. 86, 402-411. https://doi.org/10.1152/jn.2001.86.1.402 (2001).

31. Bushnell, M. C. et al. Pain perception: Is there a role for primary somatosensory cortex?. Proc. Natl. Acad. Sci. USA 96, 7705-7709. https://doi.org/10.1073/pnas.96.14.7705 (1999).

32. Mima, T., Nagamine, T., Nakamura, K. \& Shibasaki, H. Attention modulates both primary and second somatosensory cortical activities in humans: A magnetoencephalographic study. J. Neurophysiol. 80, 2215-2221. https://doi.org/10.1152/jn.1998.80.4.2215 (1998).

33. Buchner, H. et al. Differential effects of pain and spatial attention on digit representation in the human primary somatosensory cortex. NeuroReport 11, 1289-1293. https://doi.org/10.1097/00001756-200004270-00029 (2000).

34. Mennes, M. et al. Inter-individual differences in resting-state functional connectivity predict task-induced BOLD activity. Neuroimage 50, 1690-1701. https://doi.org/10.1016/j.neuroimage.2010.01.002 (2010).

35. Lorenz, J., Minoshima, S. \& Casey, K. Keeping pain out of mind: The role of the dorsolateral prefrontal cortex in pain modulation. Brain 126, 1079-1091. https://doi.org/10.1093/brain/awg102 (2003).

36. Bantick, S. J. et al. Imaging how attention modulates pain in humans using functional MRI. Brain 125, 310-319. https://doi. org/10.1093/brain/awf022 (2002).

37. Melzack, R. \& Casey, K. L. Sensory, motivational, and central control determinants of pain: A new conceptual model. Skin Sens. 1, 423-443 (1968).

38. Emerson, N. M. et al. Pain sensitivity is inversely related to regional grey matter density in the brain. Pain 155, 566-573. https:// doi.org/10.1016/j.pain.2013.12.004 (2014).

39. Erpelding, N., Moayedi, M. \& Davis, K. D. Cortical thickness correlates of pain and temperature sensitivity. Pain 153, 1602-1609. https://doi.org/10.1016/j.pain.2012.03.012 (2012)

40. Bär, K. J., de la Cruz, F., Berger, S., Schultz, C. C. \& Wagner, G. Structural and functional differences in the cingulate cortex relate to disease severity in anorexia nervosa. J. Psychiatry Neurosci. 40, 269-279. https://doi.org/10.1503/jpn.140193 (2015).

41. Geha, P. Y. et al. The brain in chronic CRPS pain: Abnormal gray-white matter interactions in emotional and autonomic regions. Neuron 60, 570-581. https://doi.org/10.1016/j.neuron.2008.08.022 (2008).

42. Kairys, A. E. et al. Increased brain gray matter in the primary somatosensory cortex is associated with increased pain and mood disturbance in patients with interstitial cystitis/painful bladder syndrome. J. Urol. 193, 131-137. https://doi.org/10.1016/j. juro.2014.08.042 (2015).

43. Ruscheweyh, R., Marziniak, M., Stumpenhorst, F., Reinholz, J. \& Knecht, S. Pain sensitivity can be assessed by self-rating: Development and validation of the Pain Sensitivity Questionnaire. Pain 146, 65-74. https://doi.org/10.1016/j.pain.2009.06.020 (2009).

44. Hu, L., Cai, M., Xiao, P., Luo, F. \& Iannetti, G. Human brain responses to concomitant stimulation of A $\delta$ and C nociceptors. J. Neurosci. 34, 11439-11451. https://doi.org/10.1523/JNEUROSCI.1355-14.2014 (2014).

45. Iannetti, G., Zambreanu, L. \& Tracey, I. Similar nociceptive afferents mediate psychophysical and electrophysiological responses to heat stimulation of glabrous and hairy skin in humans. J. Physiol. 577, 235-248. https://doi.org/10.1113/jphysiol.2006.115675 (2006).

46. Hu, L. \& Iannetti, G. Neural indicators of perceptual variability of pain across species. Proc. Natl. Acad. Sci. 116, 1782-1791. https ://doi.org/10.1073/pnas.1812499116 (2019).

47. Hu, L. et al. Was it a pain or a sound? Across-species variability in sensory sensitivity. Pain 156, 2449. https://doi.org/10.1097/j. pain.0000000000000316 (2015).

48. Hu, L., Zhang, Z., Mouraux, A. \& Iannetti, G. D. Multiple linear regression to estimate time-frequency electrophysiological responses in single trials. Neuroimage 111, 442-453. https://doi.org/10.1016/j.neuroimage.2015.01.062 (2015).

49. Beck, A. T., Steer, R. A., Ball, R. \& Ranieri, W. F. Comparison of Beck Depression Inventories-IA and-II in psychiatric outpatients. J. Pers. Assess. 67, 588-597. https://doi.org/10.1207/s15327752jpa6703_13 (2010).

50. Spielberger, C. D. State-Trait anxiety inventory. Corsini Encycl. Psychol. https://doi.org/10.1002/9780470479216.corpsy0943 (2010).

51. Jensen, M. P. The validity and reliability of pain measures in adults with cancer. J. Pain 4, 2-21. https://doi.org/10.1054/jpai.2003.1 (2003).

52. Caceres, A., Hall, D. L., Zelaya, F. O., Williams, S. C. \& Mehta, M. A. Measuring fMRI reliability with the intra-class correlation coefficient. Neuroimage 45, 758-768. https://doi.org/10.1016/j.neuroimage.2008.12.035 (2009).

53. Desikan, R. S. et al. An automated labeling system for subdividing the human cerebral cortex on MRI scans into gyral based regions of interest. Neuroimage 31, 968-980. https://doi.org/10.1016/j.neuroimage.2006.01.021 (2006).

54. Frazier, J. A. et al. Structural brain magnetic resonance imaging of limbic and thalamic volumes in pediatric bipolar disorder. Am. J. Psychiatry 162, 1256-1265. https://doi.org/10.1176/appi.ajp.162.7.1256 (2005).

55. Bi, Y. et al. 12h abstinence-induced right anterior insula network pattern changes in young smokers. Drug Alcohol Depend 176, 162-168. https://doi.org/10.1016/j.drugalcdep.2017.02.019 (2017).

56. Bi, Y. et al. Altered resting state functional connectivity of anterior insula in young smokers. Brain Imaging Behav. 11, 155-165. https://doi.org/10.1007/s11682-016-9511-z (2017).

57. Kelly, C. et al. A convergent functional architecture of the insula emerges across imaging modalities. Neuroimage 61, 1129-1142. https://doi.org/10.1016/j.neuroimage.2012.03.021 (2012). 
58. Shrout, P. E. Measurement reliability and agreement in psychiatry. Stat. Methods Med. Res. 7, 301-317. https://doi.org/10.1177/09622 8029800700306 (1998).

59. Yan, C., Wang, X., Zuo, X. \& Zang, Y. DPABI: data processing \& analysis for (resting-state) brain imaging. Neuroinformatics 14, 339-351. https://doi.org/10.1007/s12021-016-9299-4 (2016).

60. Zou, Q. et al. An improved approach to detection of amplitude of low-frequency fluctuation (ALFF) for resting-state fMRI: Fractional ALFF. J. Neurosci. Methods 172, 137-141. https://doi.org/10.1016/j.jneumeth.2008.04.012 (2008).

61. Yuan, K. et al. Amplitude of low frequency fluctuation abnormalities in adolescents with online gaming addiction. PLoS ONE 8, e78708. https://doi.org/10.1371/journal.pone.0078708 (2013).

62. Feng, D. et al. Intra-regional and inter-regional abnormalities and cognitive control deficits in young adult smokers. Brain Imaging Behav. 10, 506-516. https://doi.org/10.1007/s11682-015-9427-z (2016).

63. Xue, T. et al. Alterations of regional spontaneous neuronal activity and corresponding brain circuit changes during resting state in migraine without aura. NMR Biomed. 26, 1051-1058. https://doi.org/10.1002/nbm.2917 (2013).

64. Nichols, T. \& Hayasaka, S. Controlling the familywise error rate in functional neuroimaging: A comparative review. Stat. Methods Med. Res. 12, 419-446. https://doi.org/10.1191/0962280203sm341ra (2003).

65. Chen, X., Lu, B. \& Yan, C. Reproducibility of R-fMRI metrics on the impact of different strategies for multiple comparison correction and sample sizes. Hum. Brain Mapp. 39, 300-318. https://doi.org/10.1002/hbm.23843 (2018).

\section{Acknowledgements}

This work was supported by the National Natural Science Foundation of China (No. 31800926, 32071061, 31822025), the Project funded by China Postdoctoral Science Foundation (No. 2018M640191), the Scientific Foundation of Institute of Psychology, Chinese Academy of Sciences (No. Y8CX351005), CAS Key Laboratory of Mental Health, Institute of Psychology (No. KLMH2018ZG01), and the Magnetic Resonance Imaging Research Center, Institute of Psychology, CAS.

\section{Author contributions}

Y.Z.B. and L.H. designed the experiment. Y.Z.B. and X.H. contributed to data acquisition. Y.Z.B., J.H.Z., and L.H. analyzed the data and wrote the manuscript. All authors critically reviewed the manuscript and approved the final version for publication.

\section{Competing interests}

The authors declare no competing interests.

\section{Additional information}

Correspondence and requests for materials should be addressed to L.H.

Reprints and permissions information is available at www.nature.com/reprints.

Publisher's note Springer Nature remains neutral with regard to jurisdictional claims in published maps and institutional affiliations.

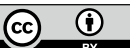

Open Access This article is licensed under a Creative Commons Attribution 4.0 International License, which permits use, sharing, adaptation, distribution and reproduction in any medium or format, as long as you give appropriate credit to the original author(s) and the source, provide a link to the Creative Commons licence, and indicate if changes were made. The images or other third party material in this article are included in the article's Creative Commons licence, unless indicated otherwise in a credit line to the material. If material is not included in the article's Creative Commons licence and your intended use is not permitted by statutory regulation or exceeds the permitted use, you will need to obtain permission directly from the copyright holder. To view a copy of this licence, visit http://creativecommons.org/licenses/by/4.0/.

(C) The Author(s) 2021 